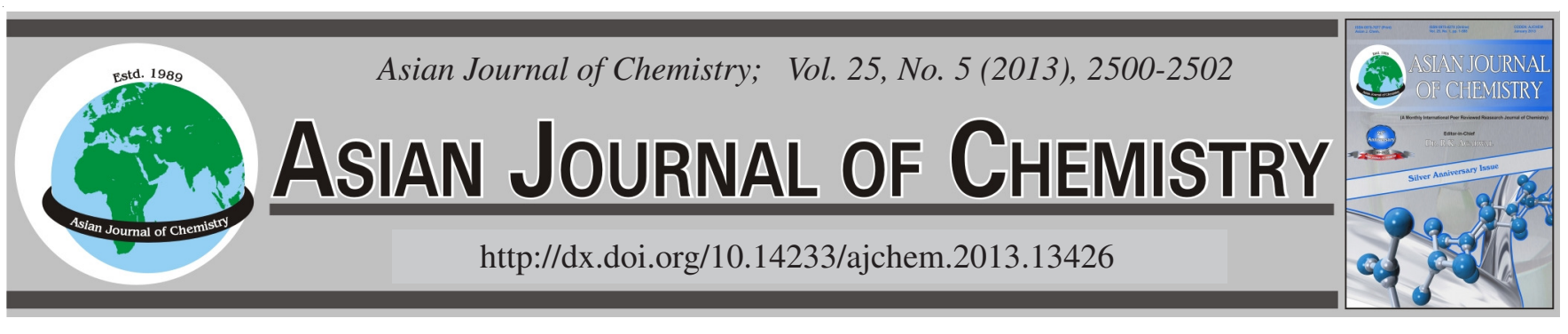

\title{
Catalase Activity Expression in Patients with Plasmodium vivax Malaria
}

\author{
R. BILGIN ${ }^{1, *}$, M.S. YALCIN ${ }^{1}$, E. KILIC ${ }^{2}$, E. KAYA ${ }^{3}$, S. YAZAR ${ }^{4}$ and I.S. KoltAS ${ }^{5}$
}

\begin{abstract}
${ }^{1}$ Department of Chemistry (Biochemistry Division), Arts and Science Faculty, Cukurova University, Adana, Turkey ${ }^{2}$ Department of Biochemistry and Clinical Biochemistry, Medical Faculty, Erciyes University, Kayseri, Turkey ${ }^{3}$ Department of Pharmaceutical Microbiology, Pharmacy Faculty, Erciyes University, Kayseri, Turkey ${ }^{4}$ Department of Parasitology, Medical Faculty, Erciyes University, Kayseri, Turkey ${ }^{5}$ Department of Parasitology, Medical Faculty, Cukurova University, Adana, Turkey

*Corresponding author: Fax: +90 322 3386070; Tel: +90 322 3386481; E-mail: rbilgin@ @ cu.edu.tr
\end{abstract}

\begin{abstract}
Plasmodium vivax (P. vivax) causes the most geographically widespread human malaria. It infects mainly the reticulocytes. Free radicals and related species have attracted a great deal of attention in recent years. They are mainly derived from oxygen and is generated in our body by various endogenous systems, exposure to different pathophysiological states. Catalase seems to be the main regulator of hydrogen peroxide metabolism. Hydrogen peroxide at high concentrations is a toxic agent, while at low concentrations it appears to modulate some physiological processes. We have analyzed the catalase levels to investigate the role of antioxidant enzymes against toxic reactive oxygen species in Plasmodium vivax infected patients and compared with healthy controls. The difference between catalase levels of patients was lower than control group and was statistically significant $(\mathrm{p}<0.05)$. In neither the patient nor control group was correlation found between age and catalase levels.
\end{abstract}

Key Words: Catalase, Malaria, Plasmodium vivax.

\section{INTRODUCTION}

Malaria is a public health problem in over 100 countries worldwide. It is a life-threatening parasitic disease transmitted by mosquitoes. The malaria parasites are protozoa of the genus plasmodium. The four species of plasmodium that cause human malaria, $P$. falciparum, $P$. vivax, $P$. ovale and $P$. malariae, are transmitted by the bite of infective female mosquitoes of the genus Anopheles. P. vivax usually does not cause life-threatening infections and only infects reticulocytes. It produces hypnozoites, which are latent in the liver. The classic clinical course of malaria consists of bouts of fever accompanied by other symptoms and alternating with periods of freedom from any feeling of illness. The intermittent type of fever is usually absent at the beginning of the disease, when headache, malaise, fatigue, nausea, muscular pains, slight diarrhoea and slight increase of body temperature are the predominant and vague symptoms, often mistaken for influenza or a gastro-intestinal infection. Most severe forms of the disease result in organ failure, delirium, impaired consciousness and generalized convulsions, followed by persistent coma and death ${ }^{1-3}$. Catalase (EC 1.11.1.6) (CAT), present in the peroxisomes nearly all aerobic cells, serves to protect the cell from the toxic effects of hydrogen peroxide by catalyzing its decomposition into molecular oxygen and water without the production of free radicals. The mechanism of catalysis is not fully elucidated, but the overall reaction is as follows:

$$
2 \mathrm{H}_{2} \mathrm{O}_{2} \rightarrow 2 \mathrm{H}_{2} \mathrm{O}+\mathrm{O}_{2}
$$

Catalase also uses hydrogen peroxide to oxidize toxins including phenols, formic acid, formaldehyde and alcohols. Peroxisomes in human are involved in the oxidation of fatty acids and the synthesis of cholesterol and bile acids. Hydrogen peroxide is a by-product of fatty acid oxidation. Antioxidant enzyme of catalase is located in the periplasmic space, which is the space between the inner and outer membranes of the cell wall. There are numerous enzymes located here that would be toxic if they were found inside the cell. The catalase found here can act on toxic molecules that are transported to the periplasm. Catalase known to be involved in functions such as protection against reactive oxygen species (ROS) generated by metabolic processes ${ }^{4-6}$.

In the present study, we aimed to characterize and investigate the role of antioxidant enzymes against toxic reactive oxygen species in human. For this purpose, we assessed antioxidant enzyme of catalase level in erythrocytes of subjects who have infected with $P$. vivax and compared them with healthy controls. 


\section{EXPERIMENTAL}

We assayed catalase activities in human serum of 135 subjects aged 3-76 years (79 males and 56 females). None were smokers, had any known pathologies and were taking steroids or medications such as iron for anaemia at the time of sampling. Serum samples for the control group were obtained from healthy people who had come to the different departments of Cukurova University/Medical Faculty for regular check-up and students or employees of the university. All subjects fasted after midnight before blood collection the next morning. In this study, 100 patients and 35 controls were examined. The mean $\pm \mathrm{SD}$ age of the patient group (infected with $P$. vivax at acute phase), which consisted of 65 men (aged $30 \pm 18$ years) and 35 women (aged $27 \pm 21$ years). The mean \pm SD age of the 14 men in the control group was $26 \pm 25$ years whilst for the 21 women was $28 \pm 20$ years.

Parasite detection: Peripheral blood samples were received from the fingers of suspected patients for malaria screening in sterile condition. Both thick and thin films preparations were made and stained with Giemsa. These prepared materials were examined for malarial parasites with 100x oil immersion objective.

Blood samples and erythrocyte extraction: Venous peripheral blood samples were collected in EDTA-coated tubes and were centrifuged at $800 \times \mathrm{g}$ for $30 \mathrm{~min}$ through a density gradient. Erythrocytes were washed three times with isotonic saline. Centrifugation for each washing was at $1400 \times \mathrm{g}$ at $4{ }^{\circ} \mathrm{C}$. Erythrocytes were then lyzed with hypotonic shocking and different dilutions were used as hemolysate ${ }^{7}$.

Determination of catalase activity: The catalase activity was measured by the method reported by Luck. One unit of catalase activity was defined as the amount of enzyme, which liberated half of the peroxidase oxygen from $\mathrm{H}_{2} \mathrm{O}_{2}$ solution in 100 at $25^{\circ} \mathrm{C}$. The enzyme activity was expressed as U/mg protein $^{8}$.

Hemoglobin concentration determination: Hemoglobin (Hb) concentrations were determined the method of White et al. ${ }^{9}$

Statistical analysis: Statistical analysis was performed with SPSS software package (Version 11.0 for Windows). Data were expressed as mean \pm standard deviation (SD). For comparison of two groups of continuous variables, independent samples $t$-test was used. A probability value of $\mathrm{p}<0.05$ indicated a statistically significant difference.

\section{RESULTS AND DISCUSSION}

Catalase scores are given in the Table-1. The difference between catalase levels of patients was lower than control group and was statistically significant $(\mathrm{p}<0.05)$. In neither the patient nor control group was correlation found between age and catalase levels.

Cells make a variety of antioxidant enzymes to fight the dangerous side effects of life with oxygen. One of the important players is catalase, which converts hydrogen peroxide into water and oxygen gas and known to be as an antioxidant enzyme. Catalase is a heme containing redox enzyme. It is produced naturally within the body and found in high concentrations in a compartment in cells called the peroxisome $e^{5,10}$.
TABLE-1

CAT LEVELS OF PATIENTS INFECTED WITH $P$. vivax AT THE ACUTE PHASE AND CONTROL GROUP

\begin{tabular}{ccccc}
\hline \multirow{3}{*}{ Group } & $\begin{array}{c}\mathrm{N} \\
\text { (female } \\
\end{array}$ & $\begin{array}{c}\text { Age (female/ } \\
\text { male) }\end{array}$ & \multicolumn{2}{c}{$\begin{array}{c}\text { CAT activity } \\
\text { (IU/mg hemoglobin) }\end{array}$} \\
& Mean \pm SD & \multicolumn{2}{c}{ Mean \pm SD } \\
\hline Patients & $100(35$ & $27 \pm 21 /$ & $15.2 \times 10^{4} \pm$ & $(\mathrm{t}=-3.01)$ \\
& $+65)$ & $30 \pm 18$ & $4.91 \times 10^{4}$ & $\mathrm{P}<0.05$ \\
\hline Controls & $35(21+$ & $28 \pm 20 /$ & $17.8 \times 10^{4} \pm$ & \\
& $14)$ & $26 \pm 25$ & $2.61 \times 10^{4}$ & \\
\hline
\end{tabular}

Catalase performs an elegant 'reshuffling' of toxic compounds. It helps the body to convert hydrogen peroxide into water and oxygen, thus preventing the formation of carbon dioxide bubbles in the blood. Catalase also uses hydrogen peroxide to break down potentially harmful toxins in the body, including alcohol, phenol and formaldehyde ${ }^{5,11}$.

Human need to oxygen to live, yet when bodies use oxygen they produce free radicals that damage cell membranes, proteins and DNA. Free radicals are chemically unstable atoms or molecules that cause other atoms and molecules in the body to become unstable as well, a process that causes extensive damage to cells and tissue and could lead to a depressed immune system, infection, cardiovascular disease, joint disease and mental decline. Free radicals are also thought to be a key component of the aging process ${ }^{5,6,9-11}$.

Malaria has been recognized as parasitic disease of humans for centuries. $P$. vivax identification is possible on the basis of the appearance of parasites of each of the four malaria species. Shape and size of asexual parasites and of macro and microgametocytes, developmental stages in peripheral blood, modifications of infected erythrocytes, presence of dots or clefts on the red blood cells are the main differential characteristics of $P$. vivax. ${ }^{1,12}$

In the human host the parasite is found primarily inside of the red blood cells (RBC). The parasite reproduces asexually inside of the red blood cell and following this the red blood cell breaks open releasing many new parasites. These parasites then infect more red blood cell's and this ultimately leads to the destruction of massive numbers of red blood cell's. The characteristic chill and fever associated with malaria occurs when the parasites are released from the red blood cell's. ${ }^{1,13}$

Because antioxidant enzymes may have an important role in the oxidant resistance of inflammatory cells, we investigated the antioxidative status of erythrocytes in patients infected with vivax malaria and compared with healthy control by measuring the one of the primary antioxidative enzyme of catalase activity. Surprisingly, we found that the catalase activity was lower in patients group than controls. At first, these data suggest that the diffusion of (unknown mechanism) $\mathrm{H}_{2} \mathrm{O}_{2}$ through membranes is lower in patients infected with $P$. vivax than that of healthy people. Another possibility for the low catalase activity is that; as it has been previously indicated ${ }^{14}$ that catalase activity might be affected by the endocytosis of the erythrocyte cytoplasm by $P$. vivax, therefore accordingly, due to haemoglobin consumption parasites might capture other proteins as antioxidant enzymes.

Lower catalase results might also show that $P$. vivaxinfected cells, including erythrocytes, produce only small 
amounts of catalase and secreted little enzyme, suggesting a lack of protective machinery against $P$. vivax. Results provide evidence for a potential role of decreased catalase in $P$. vivax by its inflammatory character. Hydrogen peroxide could not have been detoxified due to decreased activities of erythrocyte catalase. Hydrogen peroxide possibly converted to hydroxyl radical by iron due to lower transferrin level. Finally, this lower level of catalase activity could be interpreted as an indicator of malaria infection.

\section{REFERENCES}

1. B. Sina, Trends Parasitol, 18, 287 (2002)

2. N.J. White, Trends Parasitol, 18, 458 (2002).

3. L.S. Garcia and D.A. Bruckner, in ed.: S.L. Garcia and A.D. Bruckner, Diagnostic Medical Parasitology, American Society for Microbiology Press, Washington D.C., pp. 464-469 (1997).
4. T.P. Devasagayam, J.C. Tilak, K.K. Boloor, K.S. Sane, S.S. Ghaskadbi and R.D. Lele, J. Assoc. Physicians (India), 52, 794 (2004).

5. S. Muller, Mol. Microbiol., 53, 1291 (2004).

6. V.B. Djordjevic, Int. Rev. Cytol., 237, 57 (2004).

7. M.R. Waterman, Methods Enzymol, 52, 456 (1978).

8. H. Luck, in ed.: H.U. Bergmeyer, Methods of Enzymatic Analysis, Academic Press, New York, pp. 885-894 (1965).

9. W.L. White, M. Erickson and S.C. Stevens, Determination of Total Protein and Albumin Chemistry for Clinical Laboratory, The CV Mosby Company, St Louis, MO, edn. 4, p. 183 (1976).

10. M. Irshad and P.S. Chaudhuri, Indian J. Exp. Biol., 40, 1233 (2002).

11. Y.Z. Fang, S. Yang and G. Wu, Nutrition, 18, 872 (2002).

12. F.E. McKenzie and H. Bossert, J. Parasitol., 83, 593 (1997).

13. I.A Clark and W.B. Cowden, Parasitol. Today, 15, 458 (1999).

14. A. Pabon, J. Carmona, L.C. Burgos and S. Blair, Clin. Biochem., 36, 71 (2003). 